\title{
Modulation-Adaptive Cooperation Schemes for Wireless Networks
}

\author{
Yuanyuan Zhang, Yi Ma, and Rahim Tafazolli \\ Centre for Communication Systems Research \\ University of Surrey, UK, GU2 7XH. \\ Emails:\{yuanyuan.zhang, y.ma, r.tafazolli\}@surrey.ac.uk
}

\begin{abstract}
Cooperative communications can exploit the distributed spatial diversity-gain to improve the link performance. In this paper, we investigate the application of adaptive modulation concept to the decode-and-forward (DF) based cooperative network. With the relay nodes geographically close to the destination, we assume the perfect channel feedback is available only at the relay nodes, and propose a class of novel modulation-adaptive cooperation schemes (MACSs). The proposed schemes are first investigated in the single-relay scenario, and then extended to the multi-relay scenario. Simulation results show that the proposed schemes can offer the significant throughput-improvement in comparison with conventional DF systems.
\end{abstract}

\section{INTRODUCTION}

Cooperative communications [1]-[3] can exploit the distributed spacial diversity in multiuser systems to combat the impairments of the wireless channels. This is particularly useful when each node can only be equipped with a single antenna. Without the channel feedback, the conventional cooperative protocols, such as Amplify-and-forward (AF), Decode-and-forward (DF), etc, can offer a diversity gain by allowing nodes a fair opportunity to transmit messages through their own channel. On the other hand, if the channel state information (CSI) is available to the senders, the system can re-allocate the radio resource among the senders to improve the communication efficiency. Based on various cooperation protocols and network configurations, many resource allocation criteria have been proposed in [4]-[6]. The dynamic power allocations can be exploited with the full CSI available at all senders. However, it is not always practical due to the considerable feedback or processing delay. In [7], Ochiai et al. have proposed a variable-rate two-phase cooperative scheme, in which the transmission rate is determined by the geometrical properties of the network.

In this paper, we consider the scenario that the relay nodes are geographically close to the the destination, which allows the CSI available only at relays. Based on this assumption, a class of modulation-adaptive cooperation schemes (MACS) are proposed. Unlike the threshold setup for the single-link system, we establish a new threshold setup for the proposed modulation-adaptive cooperative system. Since the proposed system allows different modulation schemes for different links, the signal combining and detection at the destination rise as a new problem to be solved. These two key issues are carefully investigated. Performance analysis for the proposed system is carried out in terms of average bit error probability and average number of bits per symbol. Finally, simulation results show that the proposed scheme works well for both singlerelay and multi-relay systems. It can offer higher throughput in comparison with the conventional selective DF protocol.

\section{The Selective DF Protocol}

We first consider single relay system as shown in Fig 1, with source node $\mathrm{S}$, one relay node $\mathrm{R}$ and destination node $\mathrm{D}$. The channels among the nodes are modeled as independent slow Rayleigh fading as well as path loss.

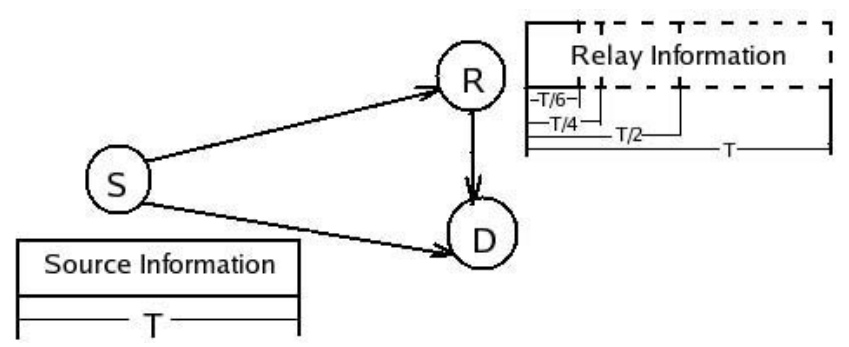

Fig. 1. A two-user transmitter cooperation model

The path loss between two nodes, for example, S and D, is modeled by

$$
P L(S, D)=K / d_{s, d}^{\alpha}
$$

where $d_{s, d}$ is the distance between the nodes, and $K$ is a constant that depends on the environment and $\alpha$ is the path loss exponent. For the free space path loss, we can choose $\alpha=2$ and $K=G_{t} G_{r} \lambda^{2} /(4 \pi)^{2}, G_{t}$ and $G_{r}$ are antenna gains at transmitter and receiver, respectively, and $\lambda$ is the wavelength [8]. Thus, if the relay is close to destination, for example, if $d_{s, d}=10 d_{r, d}$, the path loss of SD link will be $20 \mathrm{~dB}$ larger than RD link. In this paper, our discussion is based on this kind of geometric distribution of the nodes.

The instantaneous channel coefficients of SD, SR, RD link are denoted as $h_{s, d}, h_{s, r}$ and $h_{r, d}$, respectively, which are constant over a transmission block of $N$ symbols, and independent from one block to another. There is also additive independent, zero-mean complex Gaussian noise at each channel, denoted by $n_{s, d}, n_{s, r}, n_{r, d}$ with variance $\mathcal{N}_{o}$, respectively.

In the selective DF protocol, cyclic-redundancy-check (CRC) code is employed to check whether relay can get the data from source correctly. If so, relay will forward the 
data to destination in the 2 nd half of the whole transmission; otherwise, source will take the 2 nd half itself to send the same data again.

After the 1st time slot, $x_{s}$ is transmitted from S, and D and $\mathrm{R}$ get data $y_{s, d}$ and $y_{s, r}$ respectively as,

$$
\begin{aligned}
& y_{s, d}=h_{s, d} x_{s}+n_{s, d} \\
& y_{s, r}=h_{s, r} x_{s}+n_{s, r}
\end{aligned}
$$

If relay can receive the source information correctly, it will encode it to $x_{r}$ and forward to $\mathrm{D}$ in the 2 nd slot,

$$
y_{r, d}=h_{r, d} x_{r}+n_{r, d}
$$

If relay can't, using 1 bit of information, it notifies source and source re-transmits the original information itself. Then, the destination employs MRC to combine the received data for both time slots. The signal detection is performed on the output of MRC.

Generally, $x_{s}$ and $x_{r}$ are the source information, with the same coding and modulation scheme, and as shown in Fig 1, both slots will take up duration time T. However, adaptive modulation can be employed in the above model to improve the efficiency of the system. In practical communication systems, the CSI is estimated at the receivers and then fed to the transmitter, but this practical procedure results in noisy and delayed estimates being available to the transmitter. Therefore, this geometric distribution with $\mathrm{R}$ close to $\mathrm{D}$, it's more reasonable to assume $\mathrm{R}$ has the instantaneous CSI, but $\mathrm{S}$ not. The modulation-adaptive cooperation schemes for this model are proposed in the next section.

\section{Modulation AdAptive COOPERATIVE COMMUNICATIONS}

\section{A. Algorithm Description}

In the proposed scheme, a whole transmission is made up of two time slots, but the duration of the 2 nd slot is various.

Slot 1 - Information bits are modulated in low order modulation scheme, e.g., BPSK, $x_{s}$, to transmit from source. If destination can get the correct information, thanks to the CRC, the subsequent slot will be ignored, which results in the time duration for 2 nd slot equals 0 .

Slot 2 - If relay can get the data correctly at the 1st slot, it will forward the data to the destination using adaptive modulation according to the CSI of RD link. The information bits are remodulated into $x_{r}$, with modulation scheme chosen from BPSK, QPSK, 16QAM and 64QAM. The resulting slot duration will be T, T/2, T/4 and T/6 accordingly, as shown in Fig 1. Obviously, the transmission efficiency can be improved with higher order modulations. If relay can't, the source will transmit the data with duration $\mathrm{T}$.

Analogously, at the end of the 2nd slot, destination combines $y_{s, d}$ and $y_{r, d}$ together to make the final decision. For this transmission scheme, the received data from $\mathrm{S}$ and $\mathrm{R}$ may be in different modulations, thus a very important problem is how to do the combining and detection. As far as we know, no usable method has been stated. Besides, three nodes are involved in the transmission, so investigating suitable threshold becomes another important issue. In the following two subsections, these two issues will be discussed in detail.

\section{B. Detection Method}

Suppose the information bit $b$ is modulated into symbol $x_{s}$ and $x_{r}$ with different modulations. At the destination, with two received data $y_{s, d}$ and $y_{r, d}$, the a posteriori probability (APP) for the transmitted information $b$ is calculated and the maximum a posteriori probability (MAP) algorithm will be used to do the detection. The APP can be described as follows:

$$
\begin{aligned}
P\left(b \mid y_{s, d}, y_{r, d}\right) & =\frac{P\left(b, y_{s, d}, y_{r, d}\right)}{P\left(y_{s, d}, y_{r, d}\right)}=\frac{P\left(y_{s, d}, y_{r, d} \mid b\right) P(b)}{P\left(y_{s, d}, y_{r, d}\right)} \\
& =\frac{P\left(y_{s, d} \mid b\right) P\left(y_{r, d} \mid b\right) P(b)}{P\left(y_{s, d}, y_{r, d}\right)} \\
& =P\left(y_{s, d} \mid b\right) P\left(b \mid y_{r, d}\right) \frac{P(b)}{P\left(y_{s, d}, y_{r, d}\right)}
\end{aligned}
$$

The information $b$ is selected out such that the APP or $P\left(b \mid y_{s, d}, y_{r, d}\right)$ is maximized, which is mathematically represented in the following equation:

$$
\widehat{b}_{M A P}=\arg \left\{\max _{b}\left\{P\left(y_{s, d} \mid b\right) P\left(b \mid y_{r, d}\right)\right\}\right\}
$$

where the first term $P\left(y_{s, d} \mid b\right)$ is the probability to received symbol $y_{s, d}$ given the hypothesis that information bit $b$ is transmitted, and the second term $P\left(b \mid y_{s, d}\right)$ provides the extrinsic information regarding the information $b$ via the relayed symbol $y_{r, d}$.

In the case that the information bit $b$ is equally probable, the MAP equals the maximum likelihood detection as,

$$
\widehat{b}_{M L}=\arg \left\{\max _{b}\left\{P\left(y_{s, d} \mid b\right) P\left(y_{r, d} \mid b\right)\right\}\right\}
$$

In digital communications, log-likelihood is often used to simplify the calculation. For example, the log likelihood ratio of information bit $b$ with the received symbol $y_{s, d}$ can be obtained as:

$$
\begin{aligned}
\Lambda_{b}\left(y_{s, d}\right) & =\ln \left\{P\left(y_{s, d} \mid b=1\right)\right\}-\ln \left\{P\left(y_{s, d} \mid b=0\right)\right\} \\
& =\ln \left\{\sum_{M_{j} \in S_{b=1}} P\left(y_{s, d} \mid M_{j}\right)\right\}-\ln \left\{\sum_{M_{j} \in S_{b=0}} P\left(y_{s, d} \mid M_{j}\right)\right\}
\end{aligned}
$$

where, $M_{j}$ is the hypothesized modulated symbol, $S_{b=1}$ is the hypothesized set of modulated symbols whose associated information bit is $b=1, S_{b=0}$ is the hypothesized set of modulated symbols whose associated information bit is $b=0$. The noise is assumed to be complex Gaussian distributed, and thus the received symbol is the joint Gaussian random variable. The probabilities $P\left(y_{s, d} \mid M_{j}\right)$ in the above equation can be simplified to

$$
\begin{aligned}
\Lambda_{b}\left(y_{s, d}\right) & =\ln \left\{\sum_{M_{j} \in S_{b=1}} \frac{1}{\pi N_{0}} \exp \left(-\frac{\left|y_{s, d}-Q M_{j}\right|^{2}}{N_{0}}\right)\right\} \\
& -\ln \left\{\sum_{M_{j} \in S_{b=0}} \frac{1}{\pi N_{0}} \exp \left(-\frac{\left|y_{s, d}-M_{j}\right|^{2}}{N_{0}}\right)\right\}
\end{aligned}
$$


Substituting this equation to (7), the total decision is made based on,

$$
\Lambda_{b}=\Lambda_{b}\left(y_{s, d}\right)+\Lambda_{b}\left(y_{r, d}\right)
$$

\section{Adaptation Criteria}

Adaptive modulation is performed according to several SNR regions with some region boundary[9]. In this section, we discuss the region boundary for the modulation adaptation among BPSK, QPSK, 16QAM and 64QAM. Let $\gamma_{s, d}, \gamma_{s, r}$ and $\gamma_{r, d}$ denote the received SNR of SD, SR and RD link. $P_{s, d}^{b}\left(\gamma_{s, d}\right)$ (we'll use $P_{s, d}^{b}$ for simplicity) and $P_{s, r}^{b}$ denote the block error rate for SD and SR link, and $P_{s, d}$ and $P_{r, d}$ are BER of SD and RD link, respectively. If the destination can get the data correctly during the 1st time slot, the total error probability is $\left(1-P_{s, d}^{b}\right) \cdot 0=0$. If the relay can get the data correctly with the probability of $\left(1-P_{s, r}^{b}\right)$, the final errors come from the errors of the detection of combined SD and RD link, $P_{s r d}$; when the relay can't get the data correctly with the probability $P_{s, r}^{b}$, the final BER is $P_{s, d}\left(2 \gamma_{s, d}\right)$ (we use $P_{s, d}^{\prime}$ to differ it from $P_{s, d}$ with doubled SNR because of retransmission). Thus, the total BER is

$$
P_{e}=P_{s, d}^{b} \cdot\left[\left(1-P_{s, r}^{b}\right) P_{s r d}+P_{s, r}^{b} P_{s, d}^{\prime}\right]
$$

The target combining BER is denoted by $P_{\text {target }}$, and the highest order modulation will be chosen to satisfy

$$
P_{e} \leqslant P_{\text {target }}
$$

It is well known that the BER performance for $M$-QAM can be expressed as [10],

$$
P=\alpha Q(\sqrt{\beta \gamma})
$$

where $Q(x)=\frac{1}{\sqrt{2 \pi}} \int_{x}^{\infty} e^{-u^{2} / 2} d u$, and $\alpha$ and $\beta$ are parameters decided by the modulation scheme. But this equation is not easily invertible to get the appropriable modulation scheme. The block error probabilities for SD and SR link, $1-(1-$ $\left.P_{s, d}\right)^{N}$ and $1-\left(1-P_{s, r}\right)^{N}$, respectively, make the inversion of the $P_{e}$ even harder.

To simplify the adaptive modulation design and facility performance analysis, the following approximate BER expression is used [11]:

$$
B E R_{n}(\gamma) \approx a_{n} \exp \left(-b_{n} \gamma\right)
$$

For various modulations, $a_{n}$ and $b_{n}$ [9]-[11] are shown in Table I. The block error rate can also be written in the form

TABLE I

$a_{n}$ AND $b_{n}$ FOR VARIOUS MODULATIONS

\begin{tabular}{|l|l|l|l|}
\hline index & modulation & an & bn \\
\hline 1 & BPSK & 0.2 & 1 \\
\hline 2 & QPSK & 0.25 & 0.5434 \\
\hline 3 & 16QAM & 0.2 & 0.1 \\
\hline 4 & 64QAM & 0.2 & 0.0238 \\
\hline
\end{tabular}

of (14) with proper $a_{n}$ and $b_{n}$ [9]. MAP is used to perform the detection at destination, so the combined SNR is the sum of the SNRs of SD and RD link. Because the two links may not use the same modulation, the combined SNR can not be summed directly. If we write the BER expression for all modulations using $a_{1}$ and $b_{1}$ for BPSK, we get,

$$
B E R_{n}(\gamma) \approx a_{1} \exp \left(-b_{1}\left(\gamma / k_{n}\right)\right)
$$

In this way, SNR for any modulation can be equivalent to certain SNR when BPSK is used. Then, $\gamma_{E B S}=\gamma / k_{n}$ is the defined as 'equivalent BPSK SNR (EBS)', and $k_{n}$ is called 'equivalent factor'. According to Table I, we can calculate $k_{1}=1, k_{2}=2, k_{3}=10, k_{4}=42$. Thus, the BER of the combined SD and RD link is,

$$
P_{s r d}=a_{1} \exp \left(-b_{1}\left(\gamma_{s d}+\gamma_{r d} / k_{n}\right)\right)
$$

Substituting (11), (16) and (14) to (12), we obtain the region boundary SNR $\gamma_{n}$ for modulation adaptation for RD link, when $P_{s, r}^{b} P_{s, d}^{\prime} \leq P_{\text {target }} / P_{s, d}^{b}$

$$
\gamma_{n}=k_{n}\left[\frac{1}{b_{1}} \ln \left(\frac{a_{1}\left(1-P_{s, r}^{b}\right)}{\left(\frac{P_{\text {target }}}{P_{s, d}^{b}}\right)-P_{s, r}^{b} P_{s, d}^{\prime}}\right)-\gamma_{s, d}\right]
$$

where $n \leqslant 5$, and $\gamma_{1}=0, \gamma_{5}=\infty$. When the instantaneous SNR of RD link drops to the set $\left[\gamma_{n}, \gamma_{n+1}\right]$, modulation scheme with parameters $k_{n}$ will be used. If $P_{s, r}^{b} P_{s, d}^{\prime}>$ $P_{\text {target }} / P_{s, d}^{b}$, which means SD link is not reliable, to make sure the total performance can be acceptable, the lower $(1-$ $\left.P_{s, r}^{b}\right) P_{s r d}$ the better, in this case BPSK will be used at the relay to make this term as small as possible.

The scheme with SNR boundary shown in (17) is the optimal one, given name O-MACS, but seems very complicated, so we provide two simplified schemes with simpler boundary. Even though the proposed scheme can work in one of the following three modes: SD only with no error, RD and SD combined together, SD only with retransmission, the most important mode is the 2 nd one. The scheme with the target of ensuring the combining performance of SD and RD link $P_{\text {srd }} \leqslant P_{\text {target }}$, is called C-MACS. Accordingly the boundary is defined

$$
\begin{aligned}
& \gamma_{n}=k_{n}\left[\frac{1}{b_{1}} \ln \left(\frac{a_{1}}{P_{\text {target }}}\right)-\gamma_{s, d}\right](n=2,3,4) \\
& \gamma_{1}=0, \gamma_{5}=\infty
\end{aligned}
$$

when $\frac{1}{b_{1}} \ln \left(\frac{a_{1}}{P_{\text {target }}}\right)>\gamma_{s, d}$. If $\frac{1}{b_{1}} \ln \left(\frac{a_{1}}{P_{\text {target }}}\right) \leqslant \gamma_{s, d}$, which means only SD link can achieve the target BER, the 64QAM will be used at the relay.

In the above two methods, the CSI of SD link is also needed at the relay, and an easier method which does not need this information is to make sure only RD link fulfills the target, called S-MACS. If RD link fulfills the BER target, $P_{r, d} \leqslant$ $P_{\text {target }}$, the combining BER, can definitely fulfill the target. The resulting boundary region is given by

$$
\begin{aligned}
& \gamma_{n}=\frac{1}{b_{n}} \ln \left(\frac{a_{n}}{P_{\text {target }}}\right)(n=2,3,4), \\
& \gamma_{1}=0, \gamma_{5}=\infty
\end{aligned}
$$




\section{Performance Analysis}

Four modulation modes are used in the proposed scheme, and each mode $n$ will be chosen according to the SNR of the $\mathrm{RD}$ link $\gamma_{r, d}$ with probability

$$
\operatorname{Pr}(n)=\int_{\gamma_{n}}^{\gamma_{n+1}} p_{\gamma_{r, d}}(\gamma) d \gamma
$$

The instant BER of the system is shown in (11), thus the overall BER of the system $P_{e}$ also has $n$ modes $P_{e}(n)$ according to the SNR of RD link. The total average BER of the system is $\bar{P}_{e}$

$$
\bar{P}_{e}=\sum_{n=1}^{4} \int_{\gamma_{n}}^{\gamma_{n+1}} \int_{0}^{\infty} \int_{0}^{\infty} P_{e}(n) p_{\gamma_{s, d}} p_{\gamma_{r, d}} p_{\gamma_{s, r}} d \gamma d \gamma d \gamma
$$

where, $P_{e}(n)$ is $P_{e}$ in (11) with modulation scheme $n$ used at RD link, $p_{\gamma_{s, d}}, p_{\gamma_{r, d}}, p_{\gamma_{s, r}}$ are the pdf of the received SNR for SD, RD and SR link, which are with exponential distribution in Rayleigh fading channel [10].

Here, we use average bits per symbol (BPS) to evaluate the throughput of the system. The block size is $N$, if the destination can receive the data correctly at the 1 st slot, the total symbol needed is $N$; if the relay help to transmit, the total symbol needed is $N \cdot\left(1+1 / R_{n}\right)$, where $R_{n}=\log _{2}(M)$ is the carried information bits per symbol by $M$ - QAM constellation. If the relay can't help, the total symbol needed is $2 N$. Thus, the average symbol needed for transmission per bit is

$$
\begin{gathered}
\bar{S}=\sum_{n=1}^{4} \int_{0}^{\infty} \int_{0}^{\infty}\left\{\left(1-P_{s, d}^{b}\right)+P_{s, d}^{b}\left[\left(1-P_{s, r}^{b}\right) .\right.\right. \\
\left.\left.\left(1+1 / R_{n}\right) \operatorname{Pr}(n)+2 P_{s, r}^{b}\right]\right\} p_{\gamma_{s, d}} p_{\gamma_{s, r}} d \gamma d \gamma
\end{gathered}
$$

The average bits per symbol is $1 / \bar{S}$.

\section{E. Extension to multi-relay system}

Although the algorithm explained above is based on the BPSK in SD link, but the method can be extended to any modulation scheme. Meanwhile, the proposed MACS can also work in single-source multi-relay system, with model in Fig 2.

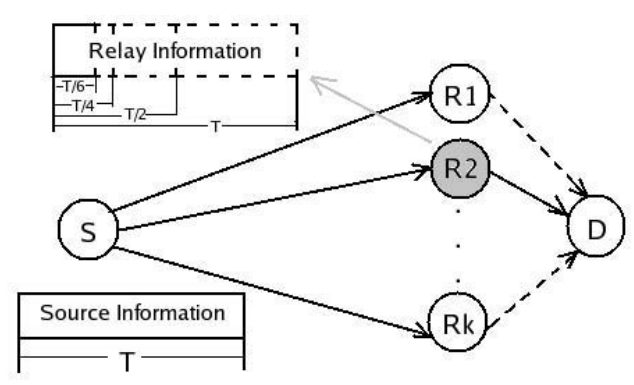

Fig. 2. single source multi relay system

In the given model, for the conventional AF/DF protocol, a total $(k+1)$ time slots will be used for the whole transmission of a block because all the relays will forward data to the destination one by one.

The adaptive modulation is exploited with relay selection. The destination will choose the relay with the highest instantaneous channel gain from the relays who receive the data correctly, to transmit with adaptive modulation in the 2nd slot. With the example in Fig 2, only the relay with solid line connected to the destination will be chosen. Thus, even multi-relays are involved in this scenario, the total transmission can be finished in at most 2 slots. The detection and adaptive modulation are performed in analog way with the single-relay system.

\section{NUMERICAL RESUlTS}

In this section, we present numerical results for the proposed system. We set the block size to $N=192$ bits, and 16-bit CRC code with generator polynomial given by coefficients 15935 (hexadecimal notation) is used. Independent flat Rayleigh fading are assumed for all channels. The SNR shown as the $X$-label is the symbol SNR for SD (the same with SR) link, ranging from $0 \mathrm{~dB}$ to $20 \mathrm{~dB}$. Relay is close to destination, resulting RD link a 20dB higher average SNR than SD and SR link, which is from 20 to $40 \mathrm{~dB}$. The target BER for adaptive modulation is set to be $1 \mathrm{e}-2$, as research in single link adaptive modulation shows with this target, the average BER of adaptive modulation is comparable with BPSK.

Single-source single-relay system Results shown in Fig 3 and 4, we compare the proposed 3 schemes with noncooperative transmission, selective DF system with CRC and two receiving antenna system. Here the selective DF simulated is modified to have CRC detection at destination for fair comparison. The comparison of cooperative schemes with non-cooperative transmission is to give a clear vision of the diversity cooperative systems can provide. Obviously, two receiving antenna system serves as a lower bound for the cooperative system. From the figures, we observe that the BER performance of the proposed system is as good as the selective DF, but the throughput has got great improvement.

Single-source multi-relay system Here we simulate the system with two relays as an example. In figure 5 and 6 , the proposed adaptive schemes with 2 relays only take at most 2 time slots, which results in a much better throughput than the selective DF, and at the same time, they achieve similar BER performance. The performance of the single relay system is also shown in the figures, and the diversity gain of the 2 relay system over the 1 relay system is quite obvious.

Based on our analysis, O-MACS should serve as the optimal scheme, but the simulation doesn't show too much advantages over the other two. Look back its SNR boundary in (17), if the $P_{s, r}^{b} P_{s, d}^{\prime}$ is larger than $P_{\text {target }} / P_{s, d}^{b}$, BPSK will be used at the relay. So, even if the $\gamma_{n}$ in (17) is quite small, the BPS performance will still be affected by the case that the source do the retransmission in the 2 nd slot. It has to pay for the low reliability of the retransmission of the source by using BPSK, which causes the decrease of average BPS. So in practical system, we can choose simpler scheme C-MACS or 


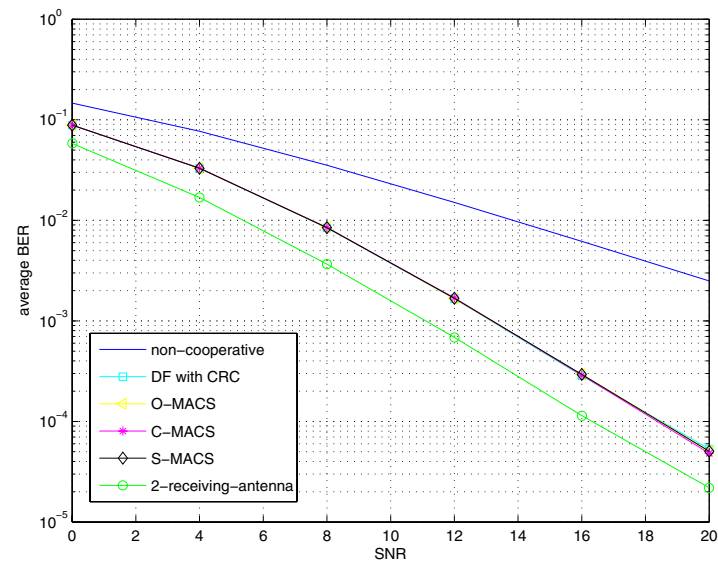

Fig. 3. BER performance for single relay system

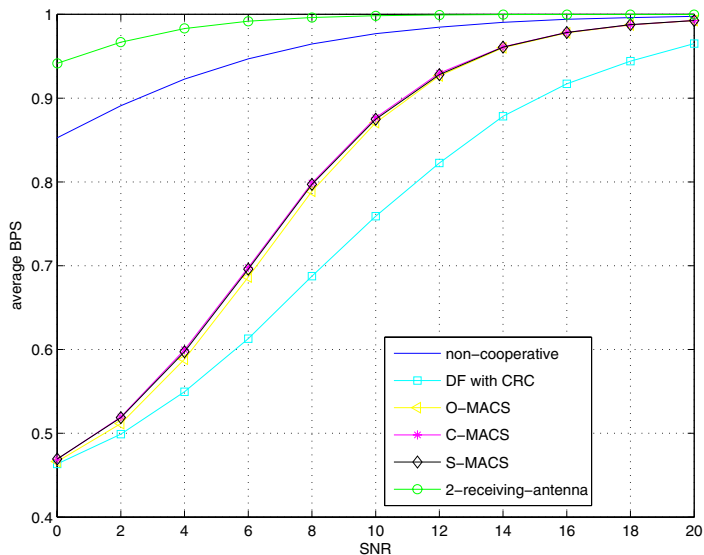

Fig. 4. Bits per symbol performance for single relay system

even S-MACS which needs less information of the channels to achieve comparable performance.

\section{CONCLUSION}

In this paper, we have proposed a class of modulationadaptive cooperation schemes for both single and multi-relay wireless communications. Requiring the CSI available only at the relay nodes, the proposed schemes could outperform the conventional DF protocol for the significant throughput improvement.

\section{REFERENCES}

[1] J. N. Laneman, D. N. C. Tse, and G. W. Wornell, "Cooperative diversity in wireless networks: Efficient protocols and outage behaviour," IEEE Transactions on Information Theory, vol. 50, no. 12, pp. 3062-3080, Dec 2004.

[2] A. Sendonaris, E. Erkip, and B. Aazhang, "User cooperation diversity - part i: System description," IEEE Transcations on Communications, vol. 51, no. 11, pp. 1927-1938, Nov 2003.

[3] —, "User cooperation diversity - part ii: Implementation aspects and performance analysis," IEEE Transcations on Communications, vol. 51, no. 11, pp. 1939-1948, Nov 2003.

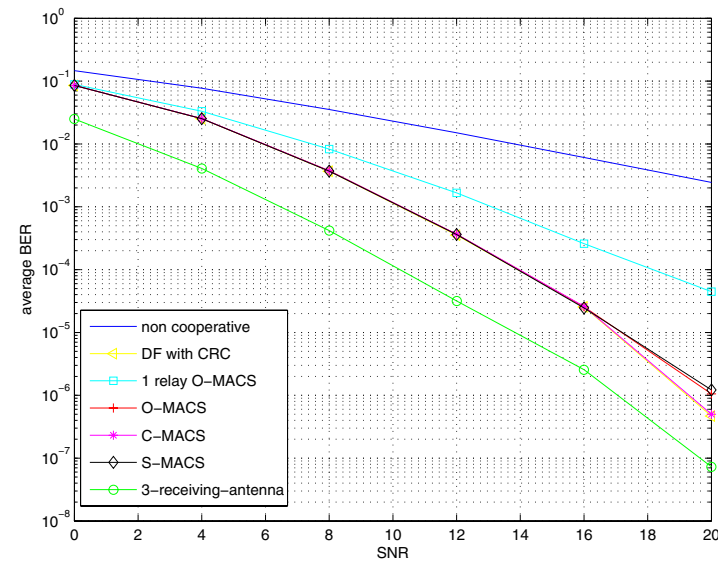

Fig. 5. BER performance for two-relay system

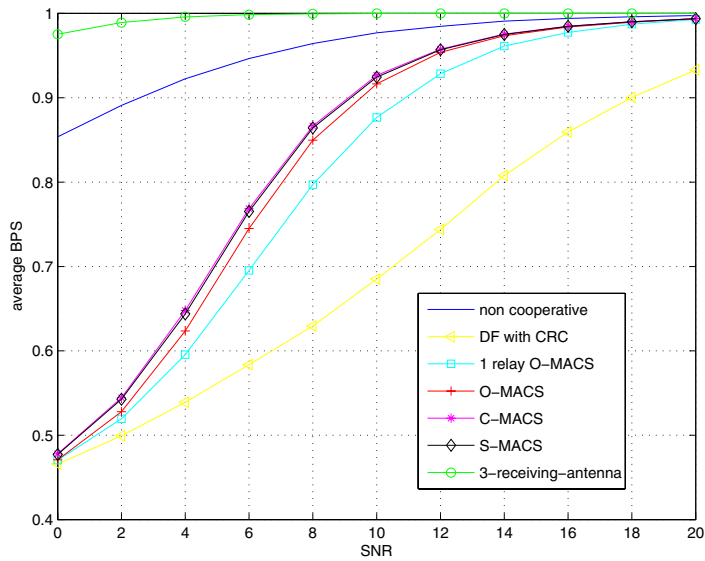

Fig. 6. Bits per symbol performance for two-relay system

[4] Y. Zhao and R. A. andT. J. Lim, "Improving amplify-and-forward relay networks: optimal power allocation versus selection," in ISIT, July 2006, pp. 1234-1238.

[5] M. Pischella and J. C. Belfiore, "Optimal power allocation for downlink cooperative cellular networks," in VTC, April 2007, pp. 2864-2868.

[6] Y. Li, B. Vucetic, Z. Zhou, and M. Dohler, "Distributed adaptive power allocation for wireless relay networks," IEEE Transaction on Wireless Communications, vol. 6, no. 3, pp. 948-958, March 2007.

[7] H. Ochiai, P. Mitran, and V. Tarokh, "Variable-rate two phase collaborative communication protocols for wireless networks," IEEE Transaction on Information Theory, vol. 52, no. 9, pp. pp.4299-4313, Sept. 2006.

[8] T. Rappaport, Wirelss communications: principle and practice, $2 \mathrm{nd}$ ed. Prentice-Hill, Englewood Cliffs, NJ, 2001.

[9] Q. Liu, S. Zhou, and G. B. Giannakis, "Cross-layer combining of adaptive modulation and coding with truncated arq over wireless links," IEEE Transactions on Wireless Communications, vol. 3, no. 5, pp. pp.1746-1755, Sept. 2004.

[10] J. G. Proakis, Digital Communications, 4th ed. McGraw-Hill, New York, 2001.

[11] M. K. Simon and M. S. Alouini, Digital communication over fading channels : a unified approach to performance analysis, 1st ed. John Wiley \& Sons, 2000.

[12] A. Goldsmith, Wireless Communications, 1st ed. Cambridge University Press, 2005. 